\title{
Multislice CT in Sinonasal Polypi; Functional Endoscopic Sinus Surgery Correlation Tarek Raslan El-Sayed ${ }^{1}$, Mohamed Fathy Ahmed Goda ${ }^{1}$, Khaled Mohamed El-Gerby ${ }^{1}$, Ezzat Ahmed Merwad ${ }^{2}$
}

Departments of ${ }^{1}$ Radiodiagnosis and ${ }^{2}$ Otorhinolaryngology, Faculty of Medicine, Zagazig University, Egypt *Corresponding Author: Tarek Raslan El-Sayed, Email: Tarekraslan2008@ gmail.com

\begin{abstract}
Background: Nasal and sinus illnesses are diagnosed with computed tomography (CT) because CT scans effectively bone anatomy is depicted with air spaces, opacified sinuses, and detailed structural details. Objective: The significance of using multislice CT for the detection and characterization of sinus polyps and the relationship between the CT findings and those of functional endoscopic sinus surgery (FESS) have been studied. Patients and Methods: At Zagazig University's Radiodiagnosis Department, we conducted this study on 18 patients, 10 of which were females and eight were males, from a total of 33 individuals. To emphasize the role of multislice (CT) in diagnosis and characterization of sinonasal polypi during functional endoscopic sinus surgery, numerous CT parameters were used and correlated with operational findings. Results: There was a strong correlation calculated by Spearman correlation coefficient (r) for rightsided and left-sided surgeries of 0.76 and 0.87 , respectively, for operative endoscopic and Lund-Mackay staging scores. Conclusion: Based on functional endoscopic sinus results, CT and diagnostic endoscopy may be the new standard of care in the diagnosis of sinonasal diseases.
\end{abstract}

Keywords: Functional Endoscopic Sinus Surgery, Multislice CT, Sinonasal Polyp.

\section{INTRODUCTION}

Sinonasal polyps can be traced back more than 4,000 years to ancient Egypt, when they were first discovered (1). In the context of the nasal cavity and paranasal sinuses, non-cancerous semi-transparent lesions are referred to as "nostril polyps." (2).

It is described by the European position paper on rhino sinusitis as an inflammation of the nose and the paranasal sinuses accompanied by two or more symptoms, as specified by the publication. Anterior or posterior nasal drip (nasal blockage, congestion, or discharge) is the most common symptom, however facial pain or pressure can also be a sign of nasal obstruction. Nasal polyps can be seen endoscopically as well as discharge that comes mostly from the middle meatus, swelling and/or mucosal blockage in the middle meatus, and/or signs of mucosal changes in the Ostiomeatal Complex (OMC) and/or sinuses on computed tomography $(\mathrm{CT})^{(3)}$.

Asthma, allergies, infections (bacterial and fungal), illnesses associated with nasal polyps, and environmental pollutants have all been connected to the development of nasal polyps ${ }^{(4)}$.

For the radiological diagnosis of nose and sinus disorders, CT scans have become the examination of choice since they vividly display air gaps, opacified sinuses, and fine structural architecture of bone anatomy. Surgeons contemplating functional endoscopic sinus surgery should use multislice CT because it can disclose anatomical structures that physical examination or diagnostic nasal endoscopy cannot ${ }^{(5)}$.

Because of its 3D high-resolution capacity, 3D highresolution $\mathrm{CT}$ is optimal for recognizing the complex anatomy and anatomic variants that are not accessible by physical examination or endoscopy. Recurrence and chronic sinus illness can now be diagnosed and managed with $\mathrm{CT}^{(\mathbf{6})}$.

Anatomical variations that may lead to difficulties before and after functional endoscopic sinus surgery (FESS) can be diagnosed with CT, which minimizes the risk of death and morbidity for patients ${ }^{(7)}$.

Functional endoscopic sinus surgery (FESS) has been widely accepted as a successful therapeutic option for medically refractory chronic rhino sinusitis (CRS) with and without sinonasal polyposis, and is the therapy of choice for patients with outstanding success rates. Improved CT techniques. The surgeon can benefit from Multidetector CT (MDCT), which has the potential to enhance diagnostic precision ${ }^{\left({ }^{(8)}\right.}$.

We aimed at this study to emphasize role of multislice (CT) in diagnosis and characterization of sinonasal polypi using various $\mathrm{CT}$ parameters and comparing CT findings to findings from functional endoscopic sinus surgery.

\section{SUBJECTS AND METHODS}

At Radiodiagnosis Department, Zagazig University Hospital, Egypt, between the months of December 2018 and December 2019, we conducted this investigation. We enrolled 18 patients in this study (10 females, 8 males) out of 33 patients, who were suspected to have sinonasal polyps. 15 cases were excluded, 2 of them were pregnant women, 3 cases were suspected to have malignant lesions, 5 uncooperative and neurotic patients, and 5 cases with extreme ages.

\section{Ethical considerations:}

As long as all participants signed informed consent forms and submitted them to Zagazig University's Research Ethics Committee, the study was allowed (ZU-IRB\#6270). We followed the World Medical 


\section{Association's ethical code for human experimentation, the Helsinki Declaration. \\ Exclusion Criteria:}

Patients with complicated sinusitis, recent nasal biopsy, with simple chronic sinusitis, previously had functional endoscopic sinus surgery (FESS) and antrochoanal polyp, patients with suspected malignant lesions, and patients with extreme ages.

\section{All patients were subjected to the following:-}

1. Complete history taking.

2. Complete general and clinical examination performed by the referring surgeon.

3. Patients were evaluated by multislice CT, and radiological findings were recorded for correlation with findings in the functional endoscopic sinus surgery (FESS).

4. Contrast material preparations: Patients who were examined with contrast material attended fasting for 6 hours. Intravenous iodinated contrast medium (60 $\mathrm{ml} / \mathrm{kg}$ urographin 76) was administrated in (5 cases) presented with suspected nasal and/or paranasal masses.

CT evaluation: All patients had undergone CT of the nose and paranasal sinuses, performed by Philips 128 MDCT in the Radiodiagnosis Department, Zagazig University Hospital. Axial and coronal cuts were taken with high resolution and slice thickness of five $\mathrm{mm}$ with bony and soft tissue windows. The results were then evaluated to provide valuable information to the surgeon regarding the nasal septal deviation, inferior turbinates hypertrophy, the presence of a concha bullosa, paradoxical turbinates, nasopharyngeal, uncinate process abnormalities, areas of dehiscence in the lamina papyracea, any of which if detected had been reported in our study. Also, the sinus opacification, how many sinuses were affected, and the severity of the sinus opacification, had been noted with the use of scoring based on the Lund-Mackay scale, which assigns a 0 for normalcy, a 1 for partial pacification and 2 for complete pacification, as well as 0,2 , or 2 for the ostiomeatal complex. Because of this, each side received a score of 24.

FESS: As a result of direct visual inspection, as well as a strong preoperative endoscopy and CT correlation, all patients were diagnosed with FESS, and the final diagnosis was based on surgical endoscopic surgery: Assessment of general endoscopic conditions was done utilising the Meltzer staging system during the procedure. A direct correlation with the discovery of each sinus on its own (fungal mud, polyp, mucous, and normal) was measured. Data from CT were reported, statistically analyzed, then compared to the data obtained from the FESS to detect points of similarity and discrepancy between them and the hypothesis test for comparing variables was conducted in order to see if there was any significant variation in abnormality ratios between CT and FESS.

\section{Statistical analysis:}

The collected data were coded, processed and analyzed using the SPSS (Statistical Package for Social Sciences) version 22 for Windows® (IBM SPSS Inc, Chicago, IL, USA). Quantitative data were presented as means, standard deviation (SD), and range. Qualitative date were presented as frequency and percentage. Spearman correlation coefficient was calculated. $P$ value 0.05 was considered statistically significant. It was judged highly significant when the $\mathrm{P}$ value was 0.001 .

\section{Results:}

The mean age of the study population was $30.96 \pm 10.98$ years old, with a range of 14 to 51 . Patients were 10 females and 8 males. Most patients aged between 20-40 years had sinonasal polypi and those with ages less than 20 years and more than 40 years were the least affected. Sinonasal polypi affected females $(55.56 \%)$ more than males $(44.4 \%)$.

Nasal obstruction was the most common complaint $(33.3 \%)$ followed by facial pain/headache $(27.8 \%)$, Nasal discharge (22.2\%), and Nasal allergy (16.7\%). Maxillary sinuses were the most commonly affected sinuses by sinonasal polypi (Figure 1)

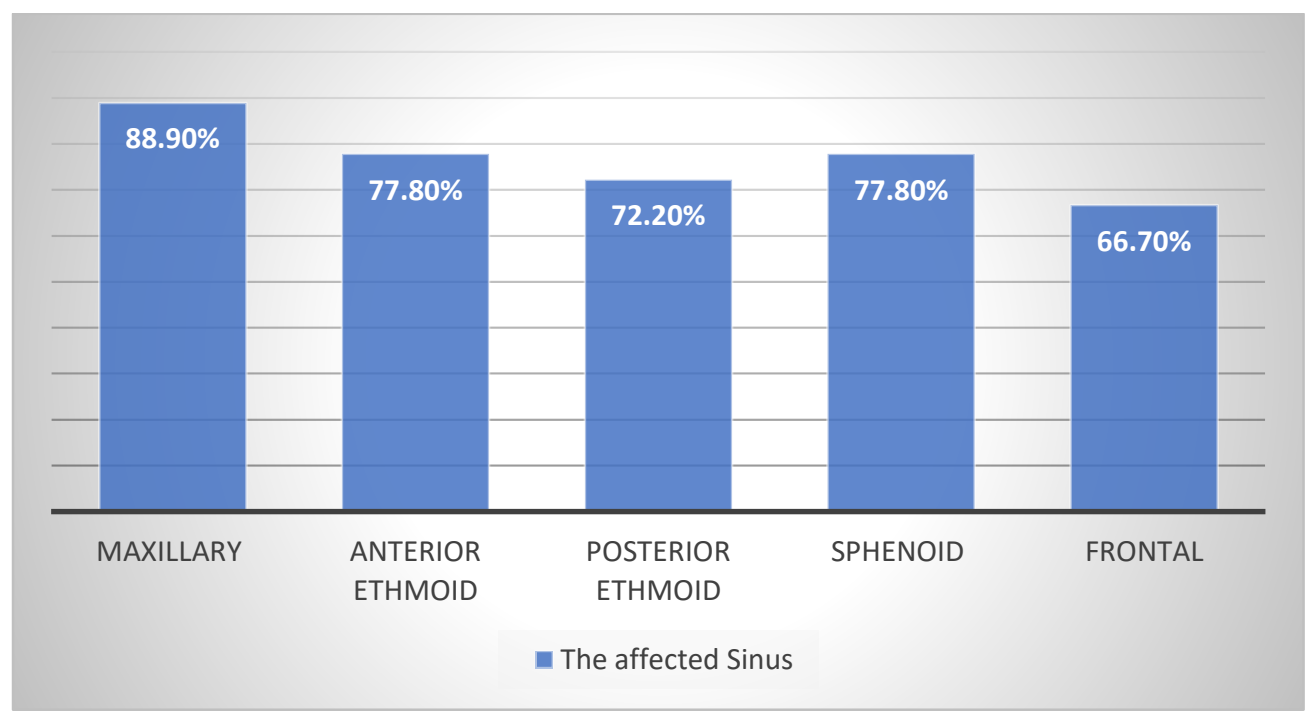

Figure (1): The affected Sinus 
The right side: Operative staging scores (Lund-Mackay scores) were found to be highly significantly correlated $(\mathrm{r}=0.76)$ with operative scores (Table 1).

Table (1): Right sided operative endoscopic and CT staging scores, and correlation between Lund- Mackay staging and operative scores on right sides results

\begin{tabular}{|l|l|l|}
\hline & Range & Mean \pm SD \\
\hline Right Lund- Mackay & $0-12$ & $1.58 \pm 0.5$ \\
\hline Right Operative & $0-4$ & $2.75 \pm 0.7$ \\
\hline & & Right Lund -Mackay \\
\hline Right Operative & r & 0.76 \\
\hline & P value & $<0.001$ \\
\hline
\end{tabular}

The left side: there was a highly significant correlation between operational staging scores and Lund-Mackay scores $(\mathrm{r}=0.87$ ) (Table 2).

Table (2): Left sided operative endoscopic and CT staging scores, and correlation between Lund- Mackay staging and operative scores on left sides results

\begin{tabular}{|l|l|l|}
\hline & Range & Mean \pm SD \\
\hline Left Lund- Mackay & $0-12$ & $1.52 \pm 0.51$ \\
\hline Left Operative & $0-4$ & $2.56 \pm 1$ \\
\hline & & Left Lund -Mackay \\
\hline Left Operative & r & 0.87 \\
\hline & P value & $<0.001$ \\
\hline
\end{tabular}

The Maxillary sinus: The results of the maxillary sinus findings revealed that $55.6 \%$ of the cases scored 2 on the Lund-Mackay scoring system. A normal sinus was discovered in 11.1 percent of the patients (Table 3).

Table (3): Maxillary sinus surgical findings in relation to CT staging score

\begin{tabular}{|c|l|c|c|c|c|}
\hline CT & FESS & \multicolumn{5}{l|}{} \\
\hline Lund-Mackay score & Normal & Discharge & Fungal mud & Polyps & Total \\
\hline 0 & $11.11 \%$ & $0.00 \%$ & $0.00 \%$ & $0.00 \%$ & $11.11 \%$ \\
\hline 1 & $0.00 \%$ & $11.11 \%$ & $5.56 \%$ & $16.67 \%$ & $33.33 \%$ \\
\hline 2 & $0.00 \%$ & $16.67 \%$ & $11.11 \%$ & $27.78 \%$ & $55.56 \%$ \\
\hline Total & $11.11 \%$ & $27.78 \%$ & $16.67 \%$ & $44.44 \%$ & $100.00 \%$ \\
\hline
\end{tabular}

The ethmoid sinuses, anterior and posterior: Anterior ethmoid sinus studies revealed that $50 \%$ of cases scored 2 on the Lund-Mackay scoring system. Polyps accounted for $38.9 \%$ of all pathology detected in the anterior ethmoid sinus. It was found that 44.4 per cent of the posterior ethmoid sinus cases scored 2 on the Lund-Mackay grading system. There were 38.9 percent of polypi (Table 4)

Table (4): Correlation of anterior and posterior ethmoid sinus CT staging scores and surgical findings

\begin{tabular}{|c|c|c|c|c|c|}
\hline CT & \multicolumn{5}{|c|}{ FESS } \\
\hline Lund-Mackay score & Normal & Discharge & Fungal mud & Polyps & Total \\
\hline 0 & $22.22 \%$ & $0.00 \%$ & $0.00 \%$ & $0.00 \%$ & $22.22 \%$ \\
\hline 1 & $0.00 \%$ & $5.56 \%$ & $5.56 \%$ & $16.67 \%$ & $27.78 \%$ \\
\hline 2 & $0.00 \%$ & $16.67 \%$ & $11.11 \%$ & $22.22 \%$ & $50.00 \%$ \\
\hline Total & $22.22 \%$ & $22.22 \%$ & $16.67 \%$ & $38.89 \%$ & $100.00 \%$ \\
\hline CT & FESS & Pon & Polyps & Total \\
\hline Lund-Mackay score & Normal & Discharge & Fungal mud & $0.00 \%$ & $27.78 \%$ \\
\hline 0 & $27.78 \%$ & $0.00 \%$ & $0.00 \%$ & $16.67 \%$ & $27.78 \%$ \\
\hline 1 & $0.00 \%$ & $0.00 \%$ & $11.11 \%$ & $22.22 \%$ & $44.44 \%$ \\
\hline 2 & $0.00 \%$ & $0.00 \%$ & $22.22 \%$ & $38.89 \%$ & $100.00 \%$ \\
\hline Total & $27.78 \%$ & $0.00 \%$ & $33.33 \%$ & & \\
\end{tabular}

Frontal sinus: The results of the frontal sinus findings revealed that $44.4 \%$ of the cases scored 2 on the Lund-Mackay scoring system. The frontal sinus was found normal in 38.9\% (Table 5). 
Table (5): Operative findings in frontal sinuses in relation to CT staging score

\begin{tabular}{|c|c|c|c|c|c|}
\hline CT & \multicolumn{5}{|c|}{ FESS } \\
\hline Lund-Mackay score & Normal & Discharge & Fungal mud & Polyps & Total \\
\hline 0 & $33.33 \%$ & $0.00 \%$ & $0.00 \%$ & $0.00 \%$ & $33.33 \%$ \\
\hline 1 & $5.56 \%$ & $0.00 \%$ & $5.56 \%$ & $11.11 \%$ & $22.22 \%$ \\
\hline 2 & $0.00 \%$ & $0.00 \%$ & $22.22 \%$ & $22.22 \%$ & $44.44 \%$ \\
\hline Total & $38.89 \%$ & $0.00 \%$ & $27.78 \%$ & $33.33 \%$ & $100.00 \%$ \\
\hline
\end{tabular}

Sphenoid sinus: The results of the sphenoid sinus findings revealed that $44.4 \%$ of the cases scored 2 . The sphenoid sinus contained polyps in $33.3 \%$ of the cases (Table 6).

Table (6): Correlation between sphenoid sinus CT staging score and surgical results

\begin{tabular}{|c|c|c|c|c|c|}
\hline CT & \multicolumn{5}{|c|}{ FESS } \\
\hline Lund-Mackay score & Normal & Discharge & Fungal mud & Polyps & Total \\
\hline 0 & $22.2 \%$ & $0 \%$ & $0 \%$ & $0 \%$ & $22.2 \%$ \\
\hline 1 & $0 \%$ & $11.1 \%$ & $11.1 \%$ & $11.1 \%$ & $33.3 \%$ \\
\hline 2 & $0 \%$ & $11.1 \%$ & $11.1 \%$ & $22.2 \%$ & $44.4 \%$ \\
\hline Total & $22.2 \%$ & $22.2 \%$ & $22.2 \%$ & $33.3 \%$ & $100 \%$ \\
\hline
\end{tabular}

An example of the findings in patients are shown in figure 2 .

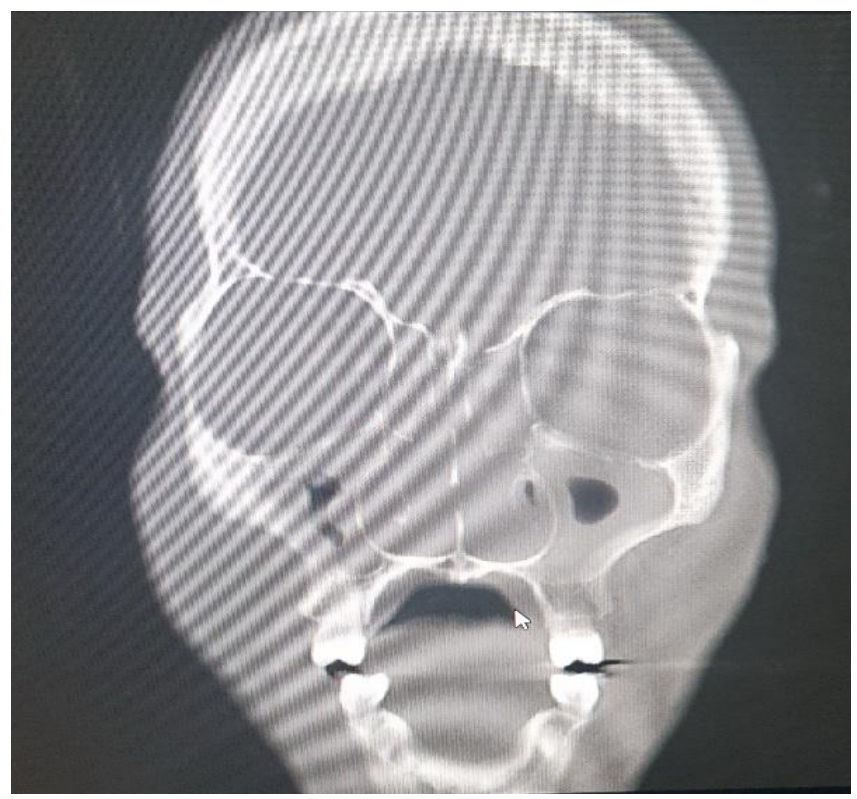

Figure (2): Female patient, 51 years old presented with headache, Persistent stuffiness and runny nose. Coronal contiguous cuts were taken through paranasal sinuses with special thin cuts for the osteomeatal complex without I.V contrast injection revealed): Near total opacification and luminal obliteration of the paranasal sinuses and nasal cavity by extensive muco-polypoidal thickening and sinonasal polyposis blending with the nasal turbinates and causing nasal blockage, Final diagnosis (FESS): Allergic pan-sinusitis with extensive sinonasal polyposis.

\section{DISCUSSION}

Prior to functional endoscopic sinus surgery (FESS), CT was regarded as the gold standard for preoperative assessment of paranasal sinuses (PNS) disorders for proper patient selection. CT scans of the
PNS and nose are required prior to planning for FESS treatment. It can serve as a "Roadmap" for the otolaryngologist to follow during surgery. Additionally, CT has a role to play in the medical-legal context ${ }^{(9,10)}$. There may be non-specific clinical signs in many situations, hence it is imperative that sinonasal polyps be evaluated by CT to rule out more aggressive infections or tumours with extra-sinus extension, osseous damage, and local invasion ${ }^{(\mathbf{1 1})}$.

The mean age of the study population was $30.96 \pm 10.98$ years old, with a range of 14 to 51. Patients between the ages of 20 and 40 were the most commonly impacted, while those between the ages of 20 and 40 were the least affected. Women were more likely than men to develop sinonasal polyps (55.56 percent). Our results are supported with study of Firoze et $\boldsymbol{a l} .{ }^{(12)}$ as they found that the mean age of the study population was $35.15 \pm 13.48$ years. In contrary to our results, a study of Sharma et al. ${ }^{(13)}$ reported that a total of 44 patients included in their study with a mean age of $40.0 \pm 17.3$ years (Range 14-71 yrs.). Male to female ratio was 1.9:1 with $29(65.9 \%)$ male and 15 (34.1\%) female.

The current study shows that regarding the right side, correlation coefficient $\mathrm{r}(0.76)$ showed a highly significant positive association between the operative endoscopic and Lund-Mackay staging scores. Correlation coefficient $r(0.87)$ was found to be highly significant between the operative endoscopic and LundMackay staging scores for the left side. Our findings are in line with those of a research by Zeid et al. ${ }^{(14)}$ which found that preoperative computed tomography (CT) and operation findings were well correlated [right (RT) side $=0.731$ and left $(\mathrm{LT})$ side $=0.814, \mathrm{P} 0<.001]$. The connection between the preoperative endoscopic and operational staging scores (RT side $=0.892$ and LT side $=0.827, \mathrm{P} 0.001)$ was likewise highly significant $(\mathrm{P}$ $0.001)$. The connection between preoperative 
endoscopic and CT staging scores was also highly significant $(\mathrm{RT}$ side $=0.768$ and $\mathrm{LT}$ side $=0.782, \mathrm{P} 0.001$ ).

Casiano ${ }^{(15)}$ reported the preoperative CT and endoscopic scores have a favourable connection. However, Stankiewicz and Chow ${ }^{(16)}$ found that the CT and preoperative endoscopic staging scores were shown to be uncorrelated, according to their study. There was no correlation between preoperative endoscopic and CT staging scores, contrary to earlier research. The participation of patients during endoscopic examinations and/or the quality of devices used for endoscopy, as well as the quality of CT pictures, could be responsible for this.

Our study found that 55.6 percent of maxillary sinus cases scored 2 on the Lund-Mackay scoring system, whereas 33.3 percent scored 1 and 11.1 percent of the cases scored 0 on the Lund-Mackay rating system. Polyps (44.4\%), discharge (27.8\%), and fungal mud $(11.8 \%)$ were the most prevalent pathologies observed in the maxillary sinus (16.7 percent). A normal sinus was discovered in 11.1 percent of the patients. Our results coincided with study of Kaku and Harugop ${ }^{\left({ }^{(17)}\right.}$ as they reported that in their study on CT scan, maxillary sinus involvement was seen in 17 patients $(56.66 \%)$ and 18 patients $(60 \%)$ on the right and left sides, respectively. Endoscopic findings of maxillary sinus secretions were present in 23 patients (76.6\%) and 24 patients (80\%), whereas edema and narrowing of sinus ostia were seen in 21 patients $(60 \%)$ and 24 patients $(80 \%)$ on the right and left side, respectively. Maxillary sinus was the second most common sinus to get affected and substantial correlation was present between CT scan and endoscopic findings.

The present study shows that the results of the anterior ethmoid sinus findings revealed that $50 \%$ of the cases scored 2 on the Lund-Mackay scoring system, whereas $27.8 \%$ scored 1 and $22.2 \%$ of the cases scored 0 . The most common pathology found within the anterior ethmoid sinus was the polyps (38.9\%), followed by the discharge $(22.2 \%)$, (16.7\%) of the cases had fungal mud and $22.2 \%$ was found to have a normal sinus. Our results agreed the results of Kaku and Harugop ${ }^{(17)}$ as they stated that on CT scan, anterior ethmoid sinus involvement was seen in 17 patients $(56.66 \%)$ and 19 patients $(63.3 \%)$ on the right and left sides, respectively. Endoscopic findings of anterior ethmoid sinus secretions were present in 19 patients $(63.33 \%)$ and 21 patients (70\%), mucosal edema was seen in 20 patients $(66.66 \%)$ and 19 patients $(63.33 \%)$, polypoidal change in sinuses in 19 patients $(63.33 \%)$ and 20 patients $(66.66 \%)$, and frank polyposis in ten patients $(33.33 \%)$ and eight patients $(26.66 \%)$ on the right and left sides, respectively. The most common pathology in anterior ethmoids was polypoidal change followed by mucosal edema, sinus secretion, and frank polyposis. Anterior ethmoid sinus was the most common sinus to be affected, and excellent correlation was present between CT scan and endoscopic findings.
The current study, shows that the results of the posterior ethmoid sinus findings revealed that $44.4 \%$ of the cases scored 2 on the Lund-Mackay scoring system, whereas $27.8 \%$ scored 0 and $27.8 \%$ of the cases scored 1. The most common pathology found within the posterior ethmoid sinus was the polyps (38.9\%), fungal mud $(33.3 \%)$ then $(27.8 \%)$ of was found to have a normal sinus. Our results coincided with reports of Chen et al. ${ }^{(18)}$, where endoscopic surgery was used to cure all instances, and it was discovered that $\mathrm{CP}$ came from the posterior ethmoidal ostium. 3 (27.3 percent) cysts, 5 (45.4 percent) polyps, and 3 (27.3 percent) mucosal edemas were seen in the posterior ethmoid sinus.

The present study shows that the results of the sphenoid sinus findings revealed that $44.4 \%$ of the cases scored 2 on the Lund-Mackay scoring system, whereas $33.3 \%$ scored 1 and finally $22.2 \%$ of the cases scored 0 . The sphenoid sinus was found normal in $22.2 \%$, with $33.3 \%$ of the cases contained polyps, $22.2 \%$ showed discharge and fungal mud in $22.2 \%$. Our results coincided with results of study of Kaku and Harugop (17) as they found that sphenoid sinus was involved on CT scan in seven patients $(23.33 \%)$ and six patients $(20 \%)$ on the right and left sides, respectively. Endoscopic finding of mucosal edema and involvement of sphenoid sinus were seen in nine $(30 \%)$ patients on the right and eight (26.66\%) patients on the left side with a moderate degree of correlation between CT scan and endoscopic findings.

\section{CONCLUSION}

There is positive correlation between functional endoscopic sinus and multislice CT findings, indicating that combination of CT and diagnostic endoscopy may become the mainstay in evaluation of the sinonasal diseases.

\section{Financial support and sponsorship: Nil.} Conflict of interest: Nil.

\section{REFERENCES}

1. Brain D, Settipane G, Lund V et al. (1997): Historical background. In: Nasal polyps. Brain, editor: epidemiology, pathogenesis and treatment. Ocean Side Publications. Berlin. Pp. 7-15.

2. Babinski D, Trawinska-Bartnicka M (2008): Rhino sinusitis in cystic fibrosis: not a simple story. Int J Pediatr Otorhinolaryngol., 72(5):619-23.

3. Fokkens W, Lund V, Mullol J (2007): European position paper on rhinosinusitis and nasal polyps 2007. Rhinology, 20: 1-136.

4. Meltzer E, Hamilos D, Hadley J (2004): Rhinosinusitis: establishing definitions for clinical research and patient care. J Allergy Clin Immunol., 114(6):155-212.

5. Fatterpekar G, Delman B, Som $P$ (2008): Imaging the paranasal sinuses: Where we are and where we are going. Anat Rec., 291(11):1564-72.

6. Ahmed M, Kanmadi S (2015). Role of computed tomography in evaluation of congenital anatomical 
variations in paranasal sinuses. Int J Biol Med Res., 6:4775-81.

7. Lund V, Savy L, Lloyd G (2000). Imaging for endoscopic sinus surgery in adults. J Laryngol Otol., 114:395-7.

8. Zojaji R, Mirzadeh M, Naghibi S (2008): Comparative evaluation of preoperative CT scan and intraoperative endoscopic sinus surgery findings in patients with chronic rhinosinusitis. Iran J Radiol., 5(2):77-82.

9. Yoon H, Kim T, Joo J et al. (2017): The impact of nasal polyposis on olfactory dysfunction in chronic rhinosinusitis. Journal of Rhinology, 24(1): 31-35.

10. Doggett S, Chino S, Lempert $T$ et al. (2018): Management of paranasal sinus metastasis by percutaneous CT-guided permanent seed brachytherapy. Practical radiation Oncology, 8(4): 221-223.

11. Nepal A, Chettri S, Joshi R et al. (2013): Benign sinonasal masses: A clinicopathological and radiological profile. Kathmandu Univ Med J., 41(1):4-8.

12. Firoze R, Kiranjith J, Sasikumar K (2019): Comparative evaluation of pre-operative MDCT findings and intraoperative ESS findings with regard to osteomeatal complex in patients with chronic rhinosinusitis. Portal Regional da BVS., 19: 1-5.
13. Sharma B, Panta O, Lohani B et al. (2015): Computed tomography in the evaluation of pathological lesions of paranasal sinuses. Journal of Nepal Health Research Council., 13: 116-120.

14. Zeid N, Kamel A, Wahba B et al. (2016): Relationship between the endoscopic, radiological and operative findings in sinonasal polyposis. Egypt J Otolaryngol., 32:147-51.

15. Casiano R (1997). Correlation of clinical examination with computer tomography in paranasal sinus disease. Am J Rhinol., 11:193-196.

16. Stankiewicz J, Chow J (2002): A diagnostic dilemma for chronic rhinosinusitis: definition accuracy and validity. Am J Rhinol., 16:199-202.

17. Kaku D, Harugop R (2017): Correlation of computed tomography and nasal endoscopic findings in chronic rhinosinusitis: A hospital-based study. Indian Journal of Health Sciences and Biomedical Research, 10: 1-6.

18. Chen F, Gao X, Yu C et al. (2008): Diagnosis and therapy of choanal polyps from posterior ethmoid sinus. Journal of Clinical Otorhinolaryngology, Head, and Neck Surgery, 22(14): 637-8. 